\title{
La Responsabilidad Social Centrada En La Accesibilidad Web
}

\section{ESJ Natural/Life/Medical Sciences}

\section{Sonia Itatí Mariño}

Facultad de Ciencias Exactas y Naturales y Agrimensura, Universidad Nacional del Nordeste, Argentina

\section{Pedro Luis Alfonzo}

Facultad de Ciencias Exactas y Naturales y Agrimensura, Universidad Nacional del Nordeste, Argentina

\section{María Viviana Godoy}

Facultad de Ciencias Exactas y Naturales y Agrimensura, Universidad Nacional del Nordeste, Argentina

Submitted: 29 August 2020

Accepted: 28 September 2020

Published: 31 October 2020

Corresponding author:

Sonia I. Mariño

DOI: 10.19044/esj.2020.v16n30p35

(c) Copyright 2020 Marino et al. Distributed under Creative Commons CC-BY 4.0 OPEN ACCESS

\section{Resumen:}

En la disciplina Informática se regulan las áreas de conocimiento y sus injerencias. Una de ellas atañe a las cuestiones profesionales y sociales, siendo la Responsabilidad Social uno de los temas. $\mathrm{La}$ universidad como agente de innovaciones en pro del sostenimiento de la sociedad, está comprometida en asegurar que sus profesionales consideren y desarrollen cuestiones vinculadas al contexto en que se desempeñan. Es así como se entiende a la Responsabilidad Social en sus connotaciones de Universitaria y Empresaria. Por otra parte, para asegurar experiencias de calidad de los ciudadanos en entornos web, uno de los aspectos a considerar es la Accesibilidad a los Contenidos Web, a través de las connotaciones tecnológicas y sociales. Se presenta un método para evaluar la Accesibilidad Web desde una perspectiva de la Responsabilidad Social Universitaria. Los datos generados a partir de la evaluación aplicada en una selección de sitios web (productos software) muestra la necesidad de fortalecer la concientización y formación continua en temas relacionados con la Accesibilidad Web, de modo de aportar con profesionales comprometidos con responsabilidad social hacia su entorno.

Subject: Computing

Palabras clave: Estándares WCAG, Accesibilidad, Accesibilidad Web, Métricas, Responsabilidad Social 


\title{
Social Responsibility Focused On Web Accessibility
}

\author{
Sonia Itatí Mariño, \\ Pedro Luis Alfonzo, \\ María Viviana Godoy,
}

Facultad de Ciencias Exactas y Naturales y Agrimensura, Universidad Nacional del Nordeste, Argentina

\begin{abstract}
In Computer Science as a discipline, these areas of knowledge and their interference are regulated. One of them concerns professional and social issues, being Social Responsibility one of the topics. The university as an agent of innovations in order to support the society is committed to ensuring that its professionals consider and develop issues related to the context in which they perform. So, the Social Responsibility definition is understood as Social and Business. So, to ensure to citizens good quality experiences in web environments, one of the aspects to consider is Accessibility to Web Content, which has technological and social connotations. In this sense, a method to evaluate Web Accessibility from a perspective of university social responsibility is presented. The data generated from the evaluation applied in web sites (software products) shows the need to strengthen the awareness and continuous training on these issues connected to Web Accessibility, in order to contribute with professionals committed to social responsibility towards their environment.
\end{abstract}

Keywords: WCAG Standard, Accessibility, Web Accessibility, Metrics, Social Responsibility

\section{Introducción}

La Accesibilidad Web (AW) aborda el acceso universal a este servicio de Internet. En este sentido, es importante que la Web sea accesible para así proporcionar un acceso equitativo e igualdad de oportunidades a las personas con diversas discapacidades; permitiendo además, que los ciudadanos participen más activamente en la sociedad (WAI, 2018; Luján Mora, 2020).

La relevancia asociada al acceso a los contenidos web, implicó a numerosos organismos internacionales en el tratamiento del tema. Entre ellos se mencionan al Consorcio WWW o W3C (2019), ISO (2020), ISO (2012), ISO (2008a), ISO (2008b), ISO (2008c), la Fundación Sidar (Sidar, 2016), el 
Centro de Investigación y Desarrollo de Adaptaciones Tiflotécnicas (Cidat, 2020) (promovido por ONCE).

Es destacable la importancia asociada a este tema, que atañe aspectos tecnológicos y sociales. En este sentido, es un tema abordado por diversos equipos de I+D+I como se infiere de las investigaciones de otros autores (Diaz et al., 2018; Mariño et al., 2018; Mariño \& Alfonzo, 2019; Amatriain et al., 2018; Lafuente et al., 2019; Barbosa et al., 2018; Monjelat et al. 2018; Sabev et al., 2020; Mariño et al., 2020). En estos trabajos se evidencia la formación en el grado y en posgrado con relación a la AW, y así se contribuye y complementa la formación curricular en temas de relevancia para la Industria del Software, sector en que se insertan los recursos humanos en que se inscribe este artículo.

En Cabrero Almenara \& Ruiz Palmero (2018, p. 20) se establecen dos posiciones en torno a las TIC e inclusión. Una de ellas trata su utilización para lograr "una educación de calidad y eliminar o disminuir con su incorporación las barreras que impiden el acercamiento de todas las personas a la educación, a la cultura y al mundo laboral". La segunda indica que en el diseño e incorporación se pueden notar "entornos y escenarios accesibles como inalcanzables”. Por lo expuesto, el segundo enfoque fundamenta la necesidad de determinar la AW en sitios con miras a aportar espacios inclusivos en la sociedad del conocimiento.

También, la informática como disciplina, dispone de diversos documentos que regulan las áreas de conocimiento y sus injerencias (Red UNCI, 2018; CONFEDI, 2018; ACM, 2019). Una de ellas atañe a las cuestiones profesionales y sociales, siendo la Responsabilidad Social (RS) uno de los temas de relevancia.

El artículo propone un método que integra aspectos de RS y AW, considerando la estrecha relación que existe entre la responsabilidad que asumen las universidades en la formación de sus futuros profesionales con la mirada sostenida en aportar a la responsabilidad social empresaria.

El artículo se estructura en cuatro secciones, donde la primera resume el marco teórico en que se inscribe el artículo, se continúa con la exposición del método, se describen los resultados y finalmente se establecen algunas conclusiones.

\subsection{Responsabilidad Social}

López Salazar et al. (2017) menciona a Puterman (2014), quien comenta entendimiento común de la Responsabilidad Social promovido por la ISO26000. En Toca Torres (2017) se define que la responsabilidad desde el punto de vista ético, implica a que todos somos responsables por todos. En González-González et al. (2017), se presenta un estudio que analiza ciertas variables que "pueden influir en la contratación de personas con algún tipo de 
discapacidad“, (p. 109) y se evidencia la responsabilidad que se asume a la sociedad.

En Torres Castaño \& Sánchez Vásquez (2014) se define la Responsabilidad Social Universitaria (RSU) como la capacidad de la universidad para "difundir y poner en práctica un conjunto de principios y valores generales y específicos, por medio de cuatro procesos claves: gestión, docencia, investigación y extensión. Así, asume su RS ante la propia comunidad tri-estamentaria y el país donde está inserta”. La universidad como agente de cambios, innovaciones y dado su alto compromiso con el sostenimiento de la sociedad, debe promover y asegurar que sus profesionales consideren y desarrollen cuestiones vinculadas al contexto en que se desempeñan. Es así como se entiende a la RSU.

En la literatura se disponen de numerosos antecedentes que tratan la RSU. Entre ellos se menciona a Larrán Jorge \& Andrades Peña (2015), quienes estudian distintas teorías de referencia para argumentar el compromiso de las universidades. Por su parte Duque \& Cervantes Cervantes (2019) tratan la temática desde un análisis bibliométrico focalizando la revisión en las bases de datos Web of Science y Scopus.

Se localizaron diversos enfoques interdisciplinarios que integran a la responsabilidad social. En Burgos Romero (2018) se relaciona con las áreas de Economía y Administración Empresarial. En Giménez (2016) se transita desde espacios de innovación curricular basados en competencias que fortalecen la formación profesional. En Alvarado Lagunas et al. (2017) se analiza la percepción en torno a las RSU en las facultades de contaduría y administración de México. Por su parte, Gaete (2015) trata la perspectiva de los significados atribuidos por los participantes internos y externos de una universidad pública española.

La Responsabilidad Social Empresaria (RSE) puede establecerse en relación a las actividades de la Industria del Software. López Salazar et al. (2017, p. 36) indican que las empresas deben implementar estrategias éticas y responsables con sus diferentes grupos de interés: socios, capital humano, clientes, comunidad, proveedores, autoridades gubernamentales y organismos. Larrán Jorge \& Andrades Peña (2015, p. 92) tratan desde la empíria el compromiso con la Responsabilidad Social Corporativa "difiere en función del tipo de organización, sector y tiempo en el que lo estemos valorando". En Toca Torres (2017) se indican algunas evidencias de la irresponsabilidad empresarial.

Los enfoques señalados precedentemente, permiten proponer un abordaje que media entre la Responsabilidad Social Universitaria y Responsabilidad Social Empresaria focalizada en la AW. 


\subsection{La accesibilidad web}

La accesibilidad web en diversos países del mundo se convirtió en una preocupación, dado que atañe directamente a la posibilidad de acceso de los ciudadanos a la información, comunicación y servicios (públicos y privados) ofrecidos a través de la WWW (Mariño \& Alfonzo, 2018; Mariño \& Alfonzo, 2019; Mariño et al, 2020; Mariño et al., 2020a).

La AW significa que los sitios web, las herramientas y las tecnologías se diseñan y desarrollan orientadas a todas las personas, asegurando que las discapacidades no sean barreras (WAI, 2018). Así, es menester contemplar a la AW desde la perspectiva de la producción de artefactos TIC y de su impacto en la sociedad.

La W3C (2019) es un organismo mundial que aborda ésta temática, siendo su función primordial desarrollar estándares para mejorar la calidad de los sitios Web y maximizar su potencial. Entre los principios que rigen en el ámbito del consorcio de W3C, se menciona lograr que los beneficios de la web estén disponibles para todos, independientemente del hardware, software, infraestructura de red, idioma, cultura, localización geográfica o habilidad física o mental (Mariño et al., 2018).

Desde la mirada tecnológica se relaciona con la Ingeniería del Software (IS), y en particular con la calidad del software. Es decir, seleccionar aquellos métodos, herramientas y procedimientos (Pressman, 2010) que permiten construir productos de calidad centrados en los principios de la WCAG 2.0 (WCAG, 2008) para presentar los contenidos y considerando las particularidades de los sujetos consumidores de la tecnología. Tambien, se trata como un requerimiento no funcional, dado que podría ser obviada desde etapas tempranas de desarrollo en algunos productos software (Mariño et al., 2012; Mariño \& Alfonzo, 2017).

Entre algunos antecedentes relacionados con la construcción de metodologías relacionadas con la AW se mencionan a López Zambrano et al. (2017), Jiménez Carreira \& Álvarez Marcos (2018) o las guías definidas por Londoño Rojas et al. (2018).

La WCAG 2.0 establece los siguientes cuatro principios teóricos: perceptible, operable, comprensible y robusto. Éstos se evalúan a través de un conjunto de pautas que se corresponden a uno o más criterios de exito y que permiten comprobar su cumplimiento o no. Tambien se considera que cada puntos de verificación de los 4 principios, se corresponde con uno de los tres niveles de prioridad establecidos por las pautas. Se establece que: i) Prioridad 1: son aquellos requisitos a cumplir para asegurar el acceso a la información de un sitio Web; ii) Prioridad 2: son aquellos puntos a cumplir, para evitar que sea muy difícil acceder a la información para ciertos grupos de usuarios; iii) Prioridad 3: son aquellos requerimientos a cumplir para evitar que ciertos usuarios experimenten ciertos problemas para acceder a la información. 
Ademas, se establecen tres niveles de conformidad asociados a las prioridades: i) Nivel de Conformidad "A": todos los puntos de verificación de Prioridad 1 se satisfacen; ii) Nivel de Conformidad "Doble A": todos los puntos de verificación de Prioridad 1 y 2 se satisfacen; iii) Nivel de Conformidad "Triple A": todos los puntos de verificación de Prioridad 1,2 y 3 se satisfacen.

\section{Metodología}

En esta sección se caracteriza el presente estudio. Dada su finalidad se trata de una investigación aplicada. Se busca construir conocimiento y validarlo en contextos específicos, en particular, la aplicación de las pautas de la WCAG 2.0 a un conjunto de sitios web que conforman el estudio primario. Según su alcance temporal, es seccional, el estudio se llevó a cabo en el año 2019, por cuestiones profesionales se mantiene el anonimato de los sitios analizados. El estudio cuantitativo se refleja en las métricas obtenidas del analisis de los resultados. El método seguido constó de las siguientes fases (Figura 1):

Fase 1. Identificación de conceptos de RS.

- Relevamiento e identificación de conceptos vinculados con la RS. Se considera un tema de relevancia atendiendo su inclusión en planes de estudios universitarios. En general este tema se encuentra contemplado entre los aspectos sociales y profesionales del profesional informático.

- Identificación de connotaciones. La RS puede entenderse en sus connotaciones de Universitaria y Empresaria. El trabajo se centró en recuperar estos aspectos contemplados desde la formación universitaria alentando a su incorporación en los futuros profesionales.

Fase 2. Evaluación de la AW con miras a su atención desde la RS.

- Selección de los objetos del estudio: Se optó por aplicar métodos primarios, es decir, se realizaron estudios sobre sitios web o artefactos software funcionales generados en el periodo 2016-2017. Se inspeccionaron 11 (once) producciones desarrolladas por estudiantes. Por razones de ética profesional no se explicitan los nombres de estos productos.

Los productos tecnológicos seleccionados se caracterizan por: i) orientación a la resolución de un problema planteado ante un requerimiento de procesamiento de la información y su difusión a través de la web; ii) utilización de entornos de desarrollo y lenguajes de libre distribución en la generación del código; iii) realización de pruebas de verificación centradas en la AW.

- Selección y descripción de las variables de la investigación relacionadas al objeto de estudio: En Londoño Rojas et al. (2018) se presenta una síntesis de guías de AW. Se optó por el estándar 
internacional WCAG 2.0 (WCAG, 2008) dada su relevancia en el orden internacional. Para obtener evidencia empírica se verificaron los principios, las pautas que éstos agrupan y los criterios comprendidos en las pautas.

- Selección de las herramientas de recolección y análisis de los datos: Se optó como herramienta el validador automático TAW, versión en línea, que automatiza la valoración de las pautas WAI 2.0. Es un validador que permite elegir el nivel de prioridad a revisar en el sitio. En el presente análisis, se optó por el nivel AA dado que es aplicado por los validadores automáticos utilizados en el estudio. Se utilizó como navegador Google Chrome.

- Ejecución de pruebas de AW automática en los sitios objetos de estudio. Se evaluaron los sitios seleccionados a través del servicio en línea proporcionado por TAW. Este evaluador de AW aplica las pautas WCAG 2.0 y brinda un informe HTML que contiene datos derivados del análisis. El resumen generado muestra el total de los problemas (indica las correcciones necesarias), las advertencias (determina aspectos que deben revisarse manualmente) y los puntos no verificados (aquellos criterios que requieren un análisis manual completo); y los organiza por cada uno de los siguientes principios: Perceptible, Operable, Comprensible y Robusto. TAW, complementariamente brinda un informe detallado del análisis realizado sobre las incidencias detectadas.

Fase 3. Diseño de estrategias para asegurar la AW desde un enfoque de RS.

- Identificadas las connotaciones que se asume desde la RS, se indagó en posibles actuaciones desde espacios de Educación Superior. La finalidad se sustenta en desarrollar diversas acciones tendientes a incorporar este aspecto de la calidad del software en la formación de los recursos humanos, dado que la AW atañe al acceso a los contenidos en las producciones tecnológicas.

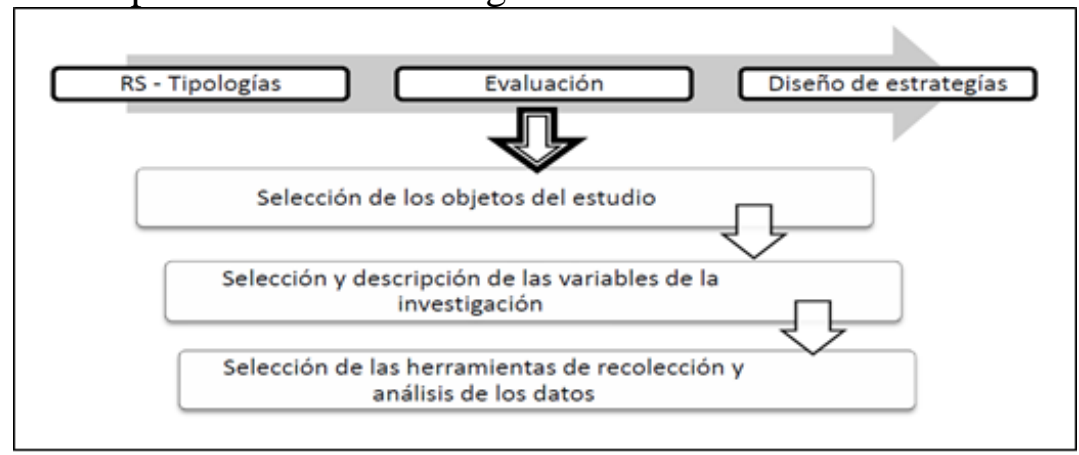

Figura 1. Método de evaluación de la AW centrada en RS. Fuente: elaboración propia. 


\section{Resultados}

En las aulas de educación superior se aborda implícitamente la RS. Sin embargo a partir del estudio empírico que se describe a continuación, se obtuvo evidencia que permite sostener la necesidad de desarrollar acciones explicitas que giren en torno a la responsabilidad social universitaria y empresaria. En el caso expuesto, se centran en el diseño y construcción de sitios accesibles que requieren implementar buenas prácticas relacionadas con las tecnologías de la información y la comunicación, particularmente aquellas vinculadas con los estándares de la AW.

Siguiendo las pautas definidas por la WCAG 2.0 (WCAG, 2008), los principios analizados se agrupan en pautas y estos a su vez contienen los criterios de éxito verificar.

A. Perceptible: son aquellas condiciones que buscan que la información y los componentes de la interfaz del usuario sean presentadas, de modo que pueda percibirse de la manera más inteligible u optima:

i. Alternativas textuales, alternativas para convertir texto a otros formatos pendiendo la capacidad de la persona que los necesite;

ii. Medios tempodependiente, para proporcionar acceso a los multimedios tempodependientes y sincronizados, como son sólo audio, sólo vídeo, audio y vídeo, audio y/o video combinado con interacción;

iii. Adaptable, contenido que pueda presentarse de diferentes formas sin perder información o estructura;

iv. Distinguible, se busca facilitar a los usuarios ver y oír el contenido, incluyendo la separación entre el primer plano y el fondo;

B. Operable: garantizar que los componentes de usuario y la interfaz de navegación deben ser fáciles:

i. Accesible por teclado, proporcionar acceso a toda la funcionalidad mediante el teclado;

ii. Tiempo suficiente, proporcionar el tiempo suficiente para leer y usar el contenido;

iii. Convulsiones, no diseñar contenido de un modo que se sepa podría provocar ataques, espasmos o convulsiones;

iv. Navegable, proporcionar medios para ayudar a navegar, encontrar contenido y determinar dónde se encuentran.

C. Comprensible: la información y el manejo de la interfaz de usuario deben ser claros. Se enfoca en características como:

i. Legibilidad, hacer que los contenidos textuales resulten claros y comprensibles;

ii. Predecible, hacer que las páginas web aparezcan y operen de manera previsible;

iii. Entrada de datos asistida, para ayudar a evitar y corregir los errores. 
D. Robusto: El contenido debe ser lo suficientemente consistente y fiable como para permitir su uso con una amplia variedad de agentes de usuario, ayudas técnicas y estar preparado para las tecnologías posteriores.

i. Compatible, para maximizar la semejanza con las aplicaciones de usuario actuales y futuras, incluyendo las ayudas técnicas.

Para ejemplificar cuestiones vinculadas a responsabilidad social empresarial enfocada en la AW, se presenta la evaluación de una muestra de 11 (once) sitios web (artefactos software funcionales), visualizándose para cada uno de ellos el número de los problemas encontrados y detectados automáticamente por la herramienta TAW, es decir, que son aplicados de manera incorrecta.

Por lo expuesto, las siguientes métricas permiten determinar el total general de problemas detectados de forma automática por cada sitio analizado (TGPS) y el total general de problemas por principio y criterio analizado (TGPP).

$$
\begin{gathered}
\text { TGPS }=\begin{array}{c}
\sum_{i=1}^{N} \text { PCA }=\text { Cantidad de criterios analizados } \\
\text { PCA }=\text { Problemas detectados por criterio analizado } \\
\mathrm{N}=\text { Cantidad de criterios analizados } \\
\text { TGPP }=\sum_{i=1}^{N} \text { PPC } \quad \text { PPP }=\text { Problemas detectados por principio y } \\
\text { criterio analizado }
\end{array}
\end{gathered}
$$

En la Figura 2 se observa la comparación de errores automáticos detectados para cada sitio analizado (TGPS) y obtenidos al evaluar la página inicial de los sitios seleccionados, con el validador de accesibilidad TAW. Los TGPS se representan en el eje Y, y corresponden a los problemas detectados automaticamente para los cuatro principios de cada sitio.

En las Figuras 3, 4, 5 y 6 se presentan los resultados derivados del análisis realizado a cada sitio web (artefactos software funcionales) por cada principio, es decir, el total general de problemas por principio y criterio analizado (TGPP). Del estudio de los valores obtenidos, se evidencia que el sitio 5 presenta mayores problemas relacionados con los 4 principios, mientras que el denominado sitio 6 presenta inconvenientes en 3 de los 4 principios.

A partir de los datos obtenidos se puede generalizar que estos productos tecnológicos requieren introducir desde etapas iniciales del proceso de software la AW como aspecto de la calidad del software. Por lo tanto, se recomienda diseñar y desarrollar considerado a la $\mathrm{AW}$ como un requerimiento no funcional y de esta manera cumplir la propuesto de la W3C. 


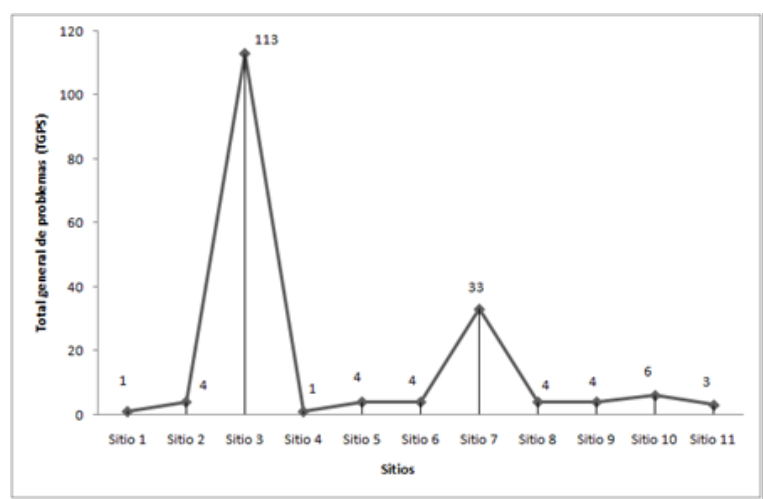

Figura 2. Métrica TGPS, comparación de problemas detectados por sitios. Fuente: elaboración propia

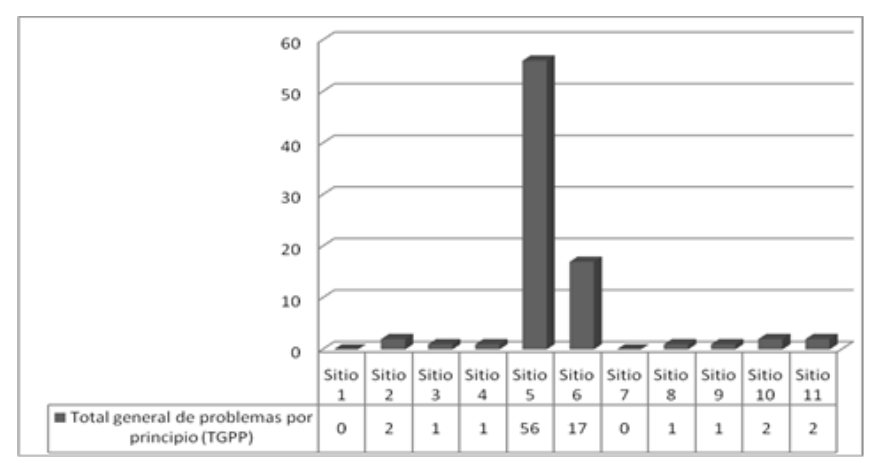

Figura 3. Métrica TGPP, comparación del incumplimiento del principio Perceptible. Fuente: elaboración propia

La métrica TGPP asociada al principio Perceptible de cada sitio que compone el análisis expresa la calidad de percepción de los usuarios respeto a los contenidos. En este sentido, los sitios del estudio con métrica cercana a 0 dan cuenta de su mayor cumplimiento del principio. La Figura 3 muestra que la mayoría de los sitios seleccionados cumplimentan este aspecto.

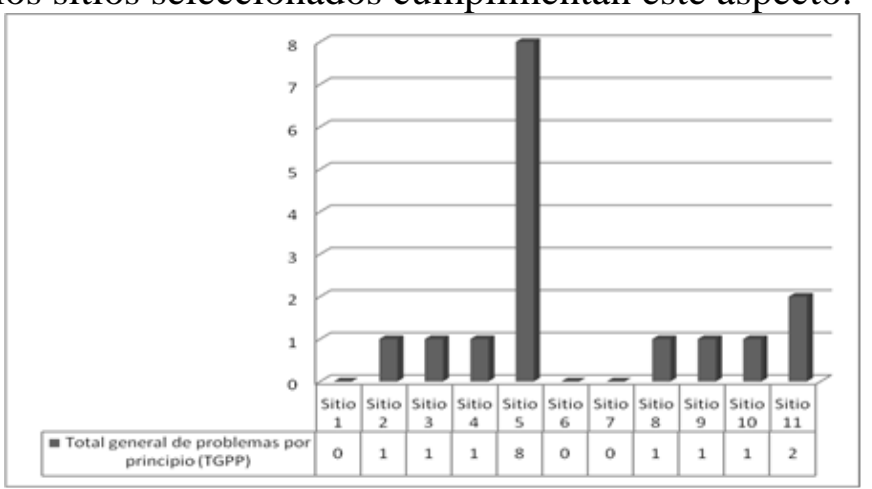

Figura 4. Métrica TGPP, comparación del incumplimiento del principio Operable. Fuente: elaboración propia 


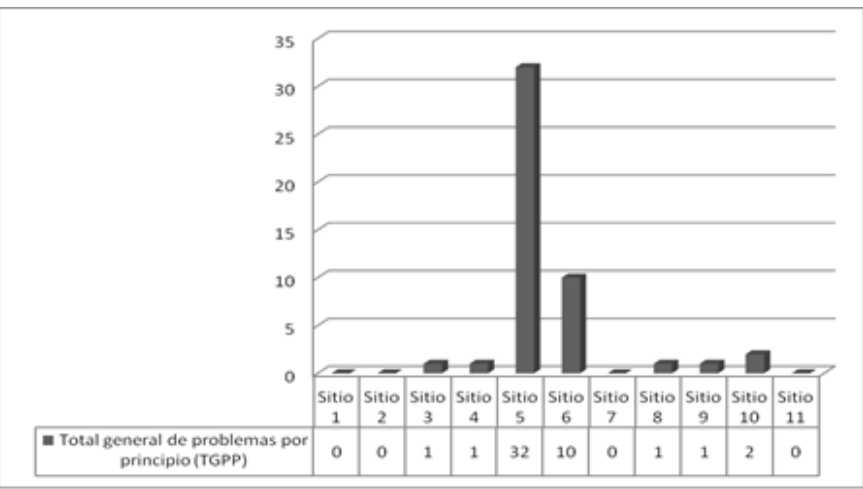

Figura 5. Métrica TGPP, comparación del incumplimiento del principio Robusto. Fuente: elaboración propia

La métrica TGPP estimada por cada sitio que compone el análisis y centrado en el principio Operable da cuenta de las diversas alternativas explicitas que se deben contemplar para asegurar las acciones o accesos a contenidos. En este sentido, los sitios del estudio con métrica cercana a 0 dan cuenta de su mayor Operabilidad, como se muestra en la Figura 4.

En referencia al principio Robusto y la métrica TGPP estimada por cada sitio que compone el análisis, el estudio aborda el respeto a las normas sobre HTML y de las hojas de estilo, para asegurar la visibilidad y acceso a los contenidos de un sitio desde cualquier navegador o dispositivo. En la Figura 5 , aquellas métricas por sitio más cercanas al 0 dan cuenta de mayor robustez del desarrollo asociado al sitio.

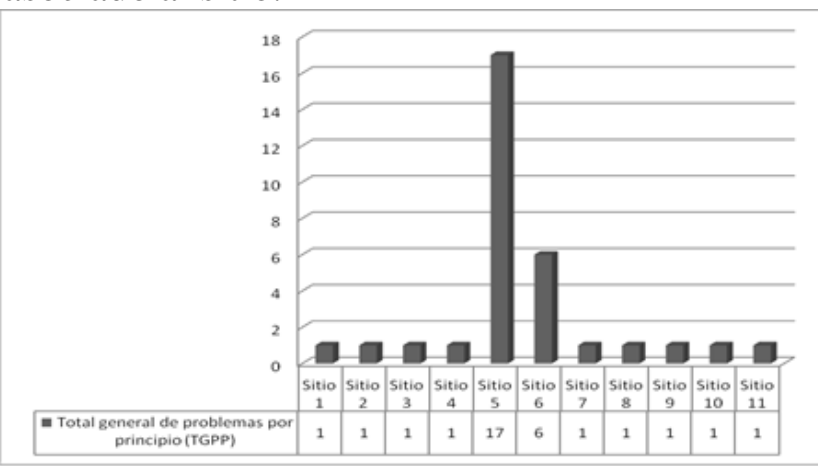

Figura 6. Métrica TGPP, comparación del incumplimiento del principio Comprensible. Fuente: elaboración propia.

En la Figura 6 se compara el cumplimiento del principio Comprensible. cuanto menor es el valor TGPP por sitio se entiende como que mejor medida se respetan aspectos de legibilidad, comprensibilidad de la forma en que se exponen los resultados y que refleja el concepto de predecible, es decir, un aspecto de facil funcionamiento del sitio. 
En Gaete Quezada (2015) se mencionan distintos enfoques para afrontar la RSU. Particularmente, los resultados del artículo se alinean con su aporte para "promover una mejor relación de la universidad con la comunidad local". Lo expuesto se sustenta en que la mayoría de los desarrollos tecnológicos que se describen aún cuando están disponibles en un entorno universal como la web, se orientan a resolver problemáticas del orden local.

En referencia a la Fase 3 del método propuesto "Diseño de estrategias para asegurar la AW desde un enfoque de RS”, se plantea:

- concientizar respecto a la importancia de la accesibilidad, y la AW en productos tecnológicos.

- diseñar capacitaciones orientadas a complementar la formación de profesionales informáticos comprometidos con responsabilidad social ciudadana.

- promover la integración de la accesibilidad web en los proyectos tecnológicos definidos en distintas asignaturas de la carrera de grado.

- promover el estudio y la adopción de buenas prácticas en el desarrollo web. Entre algunas se mencionan: señalizar el ambiente de ejecución de los desarrollos para los cuales se validan el cumplimiento de los criterios de $\mathrm{AW}$, indicar qué dispositivos y navegadores son adecuados, que herramientas adicionales se requieren para asegurar una buena experiencia de usuario, entre otros.

- propiciar la publicación de contenidos de calidad en concordancia con lo establecido por el consorcio W3C.

\section{Conclusiones}

Existen diversas cuestiones sociales, universitarias y empresariales desde las cuales se puede establecer vinculaciones con la disciplina Informática, una de ellas es la atinente a asegurar la AW a los sitios. En el artículo se presentó la AW como un aspecto desde la Ingeniería del Software dado que los productos que desde esta disciplina se generan deben ser de calidad para asegurar experiencias de usuarios positivas.

También, desde una mirada que referencia a la Responsabilidad Social Empresarial, los artefactos de las TIC deben atender cuestiones sociales por lo que sus diseños deben contemplar la resolución de problemáticas que surgen en los sujetos y para ellos.

Los datos generados a partir de la evaluación de la AW de los productos software seleccionados muestra la necesidad de fortalecer la concientización y formación continua en temas relacionados con esta temática, de modo de aportar a la formación de profesionales comprometidos con responsabilidad social al entorno que les compete. 
Numerosas herramientas y entornos de desarrollo incorporan criterios de AW. Sin embargo aun cuando los programadores adecuan el código a sus proyectos, es menester una continua e integrada revisión de estos aspectos que otorgan calidad al software destinado a todos los ciudadanos.

Como metas al corto y mediano plazo se diseñarán alternativas orientadas a complementar la formación de profesionales informáticos comprometidos con responsabilidad social ciudadana y empresaria.

\section{Agradecimientos}

El artículo se enmarca en los Proyectos de I+D+I “TI en los Sistemas de Información: modelos, métodos y herramientas" y "Sistemas informáticos. Modelos, métodos y herramientas", acreditados por la Secretaría General de Ciencia y Técnica de la Universidad Nacional del Nordeste

\section{References:}

1. ACM. (2019). Association for Computing Machinery. [On line]. https://www.acm.org

2. Alvarado Lagunas, E., Morales Ramírez, D. \& Ortiz Rodríguez, J. (2017). Un análisis sobre la percepción que los directivos y docentes tienen de la responsabilidad social universitaria en las facultades de contaduría y administración en México. Universidad \& Empresa, 19(32), 37-59.DOI: http://dx.doi.org/10.12804/http://revistas.urosario.edu.co/index.php/empr esa/article/view/4713

3. Amatriain, H., Martins, S., Bianco, S. Ribeiro, F \& Pérez. N. (2018). Herramientas de interpretación gráfica para codificación de diagramas de modelado de sistemas interpretables por un disminuido visual. XX Workshop de Investigadores en Ciencias de la Computación, Argentina.

4. Barbosa, G. \& Müller, K. (2018). Accessible content production to deaf people on the web: analysis of the video channel Ôxe. Intercom: Revista Brasileira de Ciências da Comunicação, 41(2), 153-165. https://dx.doi.org/10.1590/1809-5844201829

5. Burgos Romero, C. (2018). La Responsabilidad Social o sostenibilidad: un enfoque desde el entorno y la comunicación. RETOS. Revista de Ciencias de la Administración y Economía, 8(16), 47-59. https://dx.doi.org/10.17163/ret.n16.2018.04

6. Cabrero Almenara, J. \& Julio Ruiz Palmero, J. (2018). Las Tecnologías de la Información y Comunicación para la inclusión. Reformulando la brecha digital. IJERI: International Journal of Educational Research and Innovation. 9, 16-30.

7. CIDAT. (2020). Tecno Accesible, Centro de Investigación, Desarrollo y Aplicación Tiflotécnica. [On line]. http://cidat.once.es/ 
8. CONFEDI (2018). Consejo Federal de Decanos de Ingeniería. [On line]. https://confedi.org.ar/

9. Diaz, J. Schiavoni, A., P. Amadeo, A., Harari, I., Osorio, M. \& Carrilao, F. (2018). Análisis de aspectos de diseño en cursos masivos en línea. Caso de estudio sobre un curso de accesibilidad web. XX Workshop de Investigadores en Ciencias de la Computación (WICC), Argentina.

10. Duque, P. \& Cervantes Cervantes, L.S. (2019). Responsabilidad Social Universitaria: una revisión sistemática y análisis bibliométrico. Estudios Gerenciales, 35(153), 451-464. DOI: https://doi.org/10.18046/j.estger.2019.153.3389

11. Gaete Quezada, R. A. (2015). El concepto de responsabilidad social universitaria desde la perspectiva de la alta dirección, Cuadernos de Administración, 31(53), 97-107.

12. Gaete, R. (2015). La responsabilidad social universitaria desde la perspectiva de las partes interesadas: un estudio de caso / The university social responsibility from the perspective of the stakeholders: a case study. Actualidades Investigativas en Educación. 15. DOI 10.15517/AIE.V15I1.17729

13. Giménez, E. (2016). Responsabilidad Social Universitaria en el curriculum de la carrera de Medicina de la FCM-UNA. An. Fac. Cienc. Méd. (Asunción), 49 (1), $105-118$. http://dx.doi.org/10.18004/anales/2016.049(01)105-118

14. González-González, D., Gutiérrez-Barros. J. Ana Isabel GonzálezHerrera. A. I., Márquez-Domínguez, Y. (2017), Inserción laboral de personas con discapacidad, European Scientific Journal, 13(29), 109-125, DOI: 10.19044/esj.2017.v13n29p109

15. ISO. (2008a). ISO/IEC 9241-171:2008. Ergonomics of human-system interaction -- Part 171: Guidance on software accessibility.

16. ISO. (2008b). ISO/IEC 9241-20:2008. Ergonomics of human-system interaction -- Part 20: Accessibility guidelines or information/communication technology (ICT) equipment and services.

17. ISO. (2008c). ISO/IEC 9241-151:2008 Ergonomics of human-system interaction -- Part 151: Guidance on World Wide Web user interfaces.

18. ISO. (2012). ISO/IEC 40500:2012. Information technology - W3C Web Content Accessibility Guidelines (WCAG) 2.0. [On line]. http://www.iso.org/iso/iso_catalogue/catalogue_tc/catalogue_detail.htm? csnumber $=58625$.

19. ISO. (2020). Organización Internacional para la Estandarización. [On line]. http://www.iso.org/iso/home.html.

20. Jiménez Carreira, M. \& Álvarez Marcos, J. (2018), Metodología de análisis y evaluación de sitios web 2.0. El caso de las Diócesis Andaluzas, 
Revista de Comunicación, 17(2). http://dx.doi.org/10.26441/RC17.22018-A6

21. Lafuente, G., Ballesteros, C., Filippi, J. \& Lafuente, J. H. (2019). Implementación de una plataforma de capacitación a distancia abierta y accesible con recursos educativos destinados a personas con discapacidad visual. XXI Workshop de Investigadores en Ciencias de la Computación, Argentina.

22. Larrán Jorge, M. \& Andrades Peña, F. J. (2015). Análisis de la responsabilidad social universitaria desde diferentes enfoques teóricos. Revista Iberoamericana de Educación Superior, 6 (15), 91-107.

23. Londoño Rojas, L. F., Tabares Morales, V., Rosecler Bez, M. \& Duque Mendez, N. D. (2018). Análisis comparativo de guías para el desarrollo web accesible. Ciencia e Ingeniería Neogranadina, 28 (1), 101-115. DOI: http://dx.doi.org/10.18359/rcin.2683

24. López Salazar, A., Ojeda Hidalgo, J. F. \& Ríos Manríquez, M. (2017). La responsabilidad social empresarial desde la percepción del capital humano. Estudio de un caso. Revista de Contabilidad, 20(1), 36-46.

25. López Zambrano, J., Moreira Pico. J. \& Alava Cagua, N. (2017) Metodología para valorar y clasificar herramientas de evaluación de accesibilidad web. e-Ciencias de la Información, 8(1).

26. Luján Mora, S. (2020). Accesibilidad Web, Definición de accesibilidad web. [On line]. http://accesibilidadweb.dlsi.ua.es/?menu=definicion

27. Mariño, S. I., Godoy, M. V., Alfonzo, P. L., Acevedo, J., Gómez Solís. L. \& Fernández Vázquez, A. (2012). Accesibilidad en la definición de requerimientos no funcionales. Revisión de herramientas. Revista Multiciencias, 12(3), 305-312.

28. Mariño S. I. \& Alfonzo, P. L. (2017). Evaluación de la accesibilidad web. Una mirada para asegurar la formación en la temática. Campus Virtuales, 6(2), pp. 21-30.

29. Mariño S., Alfonzo, P., Galain, C., Maidana, J. \& Alderete, R. (2018). Accesibilidad web, aportando a la inclusión. XX Workshop de Investigadores en Ciencias de la Computación, Argentina

30. Mariño S. I. \& Alfonzo, P. L. (2019). Evidencias de Accesibilidad Web en la generación de sitios. Propuesta de un método. Revista Iberoamericana de Tecnología en Educación y Educación en Tecnología, 23, 52-60. DOI: 10.24215/18509959.23.e0

31. Mariño, S. I., Alfonzo, P. L. \& Godoy, M. V. (2020). Medidas de accesibilidad web. Aplicación en una plataforma educativa. European Scientific Journal, 16(1), 11-22, http://dx.doi.org/10.19044/esj.2020.v16n1p11 
32. Mariño, S. I., Alfonzo, P. L. \& Godoy, M. V. (2020a). Accesibilidad Web. Un aporte de responsabilidad social universitaria. En Ed. Atena, DOI 10.22533/at.ed.6832020032

33. Monjelat, N. G., Cenacchi, M. A. \& San Martín, P. S. (2018). ¿Programación para Todos? Herramientas y Accesibilidad: Un Estudio de Caso. Revista latinoamericana de educación inclusiva, 12(1), 213-227. https://dx.doi.org/10.4067/S0718-73782018000100213

34. Pressman, R. S. (2010). Ingeniería de Software: Un Enfoque Práctico. Madrid: Pearson Education, S.A.

35. Puterman, P. (2014). ¿'Se agotó la RSE?, Diario responsable. [On line]. https://diarioresponsable.com/opinion/18664-perla-puterman

36. Red UNCI (2018). Redes Universitarias de Carreras de Informática. [On line]. http://redunci.info.unlp.edu.ar/

37. Sabev, N., Georgieva Tsaneva, G., \& Bogdanova, G. (2020). Research, analysis, and evaluation of web accessibility for a selected group of public websites in Bulgaria. Journal of Accessibility and Design for All. 10(1), 124-160. DOI: 10.17411/jacces.v10i1.215

38. Sidar. (2016). Fundación Sidar, Fundación Sidar - Acceso Universal-. [On line]. http://www.sidar.org/

39. TAW. Validador de accesibilidad web. [On line]. https://www.tawdis.net

40. Toca Torres, C. E. (2017). Aportes a la responsabilidad social. Revista Mexicana de Ciencias Políticas y Sociales. LXII, 230, 393-408.

41. Torres Castaño, A. G. \& Sánchez Vásquez, L. M. (2014). La responsabilidad social universitaria desde su fundamentación teórica. Libre Empresa. 21, 69-105.

42. WAI (2018). Web Accessibility Initiative. WAI. Introduction to Web Accessibility.https://www.w3.org/WAI/fundamentals/accessibilityintro/\#what.

43. WCAG. (2008). Web Content Accessibility Guidelines (WCAG) 2.0. [On line]. http://www.w3.org/TR/WCAG20/

44. W3C. (2019). About W3C. [On line].https://www.w3.org/Consortium/ 